\title{
Design Method of the Measuring System for the Mirror Surface Spacing of a compound lens
}

\author{
Ping Zhong ${ }^{1, a}$,Shaohui Pan ${ }^{1, b}$, Zhisong $\mathrm{Li}^{2, \mathrm{c}}$ and Xingyu Gao ${ }^{1, \mathrm{~d}}$ \\ ${ }^{1}$ College of Science, Donghua University, Shanghai 201620, PR China \\ ${ }^{2}$ College of Information Science and Technology, Donghua University, Shanghai 201620, PR China \\ apzhong937@dhu.edu.cn, ${ }^{\mathrm{b}}$ 1571565468@qq.com, ${ }^{\mathrm{c}}$ 272808216@qq.com, ${ }^{\mathrm{d}}$ 1019098097@qq.com
}

Keywords: Optical fiber low coherence interference, lens mirror space, Signal-to-noise ratio, Band-pass filter, least square method

Abstract: Based on the principle of low coherent light interference of optical fiber, a new method for detecting the distance between the mirror surfaces of a compound lens is proposed. Firstly, a system based on the principle of low coherent light interference is designed. Secondly, the frequency calculation method of interference signal of measurement system is put forward, and the band-pass filter is designed for interference signal to improve the signal-to-noise ratios. Based on the characteristics of low coherence light interference signal, the least squares symmetric peak location algorithm is proposed, which realizes the precise locating of the peak of the interferometric signal. Besides, the experiment of measuring the distance between the compound lens is carried out and the measurement error is analyzed.

\section{Introduction}

In the manufacture of optical instruments, the center thickness machining precision and assembly precision of the lens have an important influence on the imaging quality of the optical system. Especially in high-performance precision optical systems such as aviation, aerospace and microscope systems, there are strict control requirements for the lens center thickness and the mirror spacing. So, how to get high precision measurement results is a challenging topic. At present, the method for measuring distance between the mirror surfaces of a compound lens is mainly divided into two types, the one is contact measurement and the other is non-contact measurement. The structure of contact measurement is relatively simple, but it has a large defect itself, such as easy to scratch the lens and destroy the lens. The frequent contact between the surfaces of the probe and lens can also make the worn lenses and affect the measurement accuracy. Non-contact measurement system is relatively complex, but it can perform nondestructive testing on the lens group. It is mainly to measure the relative position of the mirror surface by measuring the reflection signal of the lens surface. The methods mainly include image method ${ }^{[1]}$, axial dispersion ${ }^{[2]}$, differential confocal method ${ }^{[3]}$, image calibration method $^{[4,5]}$, etc. Low-coherence optical interferometry, as the main detection method of non-contact measurement for optical devices, is one of the hotspots today. In this paper, based on the principle of low-coherence light interference, a system for measuring the lens spacing of the compound lens is designed and the main factors affecting the detection accuracy are studied, and the effective method for signal filtering and waveform peak location is proposed.

\section{Measurement principle and system design of lens spacing}

Low-coherence light interferometry ${ }^{[6-12]}$ is an interferometric technique using a broad-spectrum light source as a coherent light source. The coherence length is short and the interference peak can be generated only when the optical path of the measured light and the reference light is equal, so it has a good spatial positioning characteristic. By calculating the distance between the interference peaks at the apex of the front and rear mirror surfaces, the lens group spacing and the center thickness of the lens can be calculated. In this paper, the basic structure of Michelson interferometer is adopted, in which the light emitted the light source is passed through the optical fiber coupler to produce the measured light and the reference light respectively. On the one hand, the reflected signal come from the different surfaces of the sample has a different optical path. On the other hand, the reference 
optical path will change with the position of the reference mirror. Only the reflected signal from a specific location of the sample can be coherent with the reference light, where the location of the maximum intensity of coherent signal corresponds to the equal optical path position. The structure of the lens spacing measurement system is designed as shown in Fig.1.

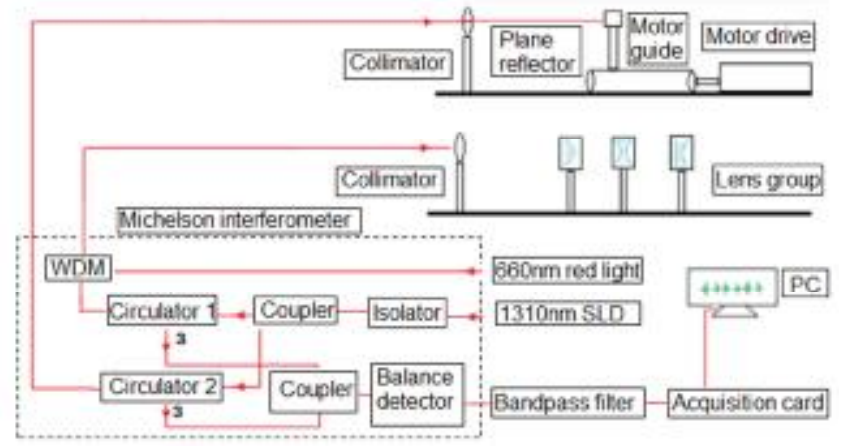

Fig.1. Lens group spacing detection system diagram

The light emitted from the wide-spectrum light source SLD (center wavelength $1310 \mathrm{~nm}$, half-wave width $85 \mathrm{~nm}$ ) is split by the fiber coupler and then enters the circulator 1 and the circulator 2 , and forms measurement light and reference light, respectively. In order to facilitate the installation and debug the detection system, the visible light with the wavelength of $660 \mathrm{~nm}$ and the measured light are mixed in the wavelength division multiplexer, and then, they are projected onto the front and back surfaces of the lens group by the collimator and reflected by the front and back surfaces of the lenses in the compound lens. Finally, the reflected light is shot into the ring through the collimator and the wavelength division multiplexer and recoupled into the optical fiber system from the port 3 . In the same way, the reference light is reflected by a movable plane reflector. The returned reference light is ejected through port 3 of the ring and interferes with the measured light in the optical fiber coupler. The returned reference light is ejected through port 3 of the ring and interferes with the measured light in the optical fiber coupler. The path of the reference light can be changed by driving a plane reflector to move uniformly in a straight line. When the optical path of the measured light is equal to that of the reference light, that is, when the optical path difference is zero, the interference signal is strongest, at this time the maximum wave peak signal can be detected by the computer.

Since the measuring beam contains many light rays reflected from the front and rear surfaces of multiple lenses, there will be multiple sets of maximum wave peaks in the process of moving plane reflector. According to the relative position of two zero light path difference, the measurement of lens spacing of compound lens can be completed. In the process of measurement, the information of the plane reflector is recorded in real time by a high resolution grating scale displacement sensor. In order to suppress the common-mode noise of the system and improve the signal-to-noise ratio of the system as much as possible, the system proposed in this paper uses a balanced detector for the photoelectric conversion of the interference signal. Then the data acquisition card is controlled by the computer, and the signal is processed by the computer. In order to further suppress the noise, a signal filter is designed.

\section{Signal filtering and waveform peak location}

\section{Design of band-pass filter}

In the detection system, the intensity of reflected light of the plane mirror is higher than the intensity of the tested lens. When the intensity ratio of the incident light passing through the reference light and detection light is 50:50, the interference signal is very weak. Fig.2(a) shows the waveform of the interference signals. In this case, the peaks of interference signals are difficult to extract. Through experiments, we can set the intensity ratio of the incident light passing through the reference light and detection light as 80:20, and the output interference signals are very obvious as shown in Fig.2(b). 

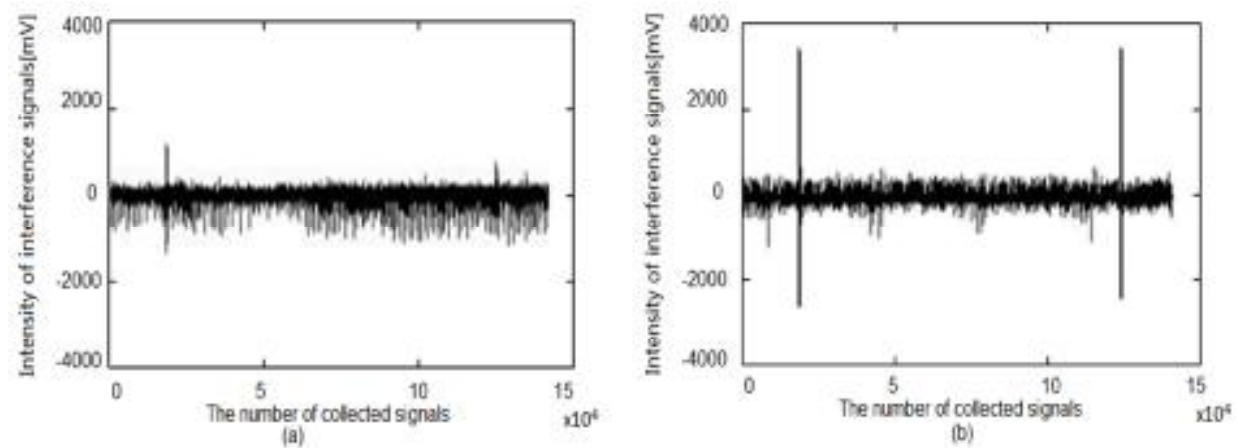

Fig.2. The comparison of interference signal intensity.(a) the waveform of the interference signals while the intensity ratio is 50:50; (b) the waveform of the interference signals while the intensity ratio is 80:20

It can be seen from Fig.2 (b) that, although the strong contrast interference signal can be obtained by setting the light intensity ratio between the reference light and detection light, the interference signal are often mixed with noise, which directly affects the location of peak signal. In this paper, a band-pass filter is designed to suppress the noise signal and improve the signal-to-noise ratio $^{[13,14]}$. The center wavelength of the designed filter can be calculated. Assuming that the moving speed of the plane mirror on a guide rail is $v_{\text {mirror }} \mathrm{mm} / \mathrm{s}$ during detection process and the center wavelength of the broadband light source is $\lambda_{0} \mathrm{~nm}$, then the center wavelength $\left(f_{0}\right)$ of the filter can be calculated by the Eq.(1):

$$
f_{0}=\left(2 v_{\text {mirror }} / \lambda_{0}\right) \times 10^{6} \text {. }
$$

For the system designed in this paper, $\mathrm{v}_{\text {mirror }}$ is $3.78 \mathrm{~mm} / \mathrm{s}$, the wavelength rang of the broadband light source is $1290 \sim 1330 \mathrm{~nm}, \lambda_{0}$ is $1310 \mathrm{~nm}$, thus the $f_{0}=5.77 \times 10^{3} \mathrm{kHz}$ and the frequency range of a band-pass filter is from $4.77 \mathrm{kHz}$ to $6.77 \mathrm{kHz}$, and the center frequency can be selected as $5.77 \mathrm{kHz}$.

\section{Method for locating the peak of interference signal}

In this paper, the location of the peak of interference signal is the key to detection of lens spacing and can be determined by extracting the signal envelope. According to the principle of low coherence interference, the wave peak of interference signal represents the interference generation between the reference light and detection light at zero optical paths. For measuring system, as long as the optical path difference between the two peaks of the interferometric signal from the lens group is obtained, the distance between the lenses of compound lens can be obtained. A simple method is to use the peak signal to determine the location of the zero optical path difference. However, because the adjacent extreme signals are so close to each other, the noise introduced in the detection process can easily cause location error which will lead to poor repeatability accuracy of the measurement system. The Gauss fitting algorithm has the advantage of locating peaks, but it requires that the interference signal has good symmetry. So it cannot meet the requirements of the system designed in this paper. In this paper, a symmetric peak location algorithm based on the least square method is proposed.

First, the acquired interference signals are preprocess. Fig.3(a) shows the primary signals collected. The position of the mean value of the intensity is set to the abscissa axis. Fig.3(b) shows the sum of the absolute values for the same position signals. The envelope image of the signals is represented by a dotted line shown in Fig.3(b).

According to the theory of partial coherence, when the value of function of the location points in envelope curve is greater than 1/e of the maximum value, these points are directly related to the coherence length of the light source. So the signals whose value is greater than 1/e of the maximum value point are selected as an effective processing signal, and then the location of peak signal can be determined by the a symmetric peak location algorithm based on least square. 


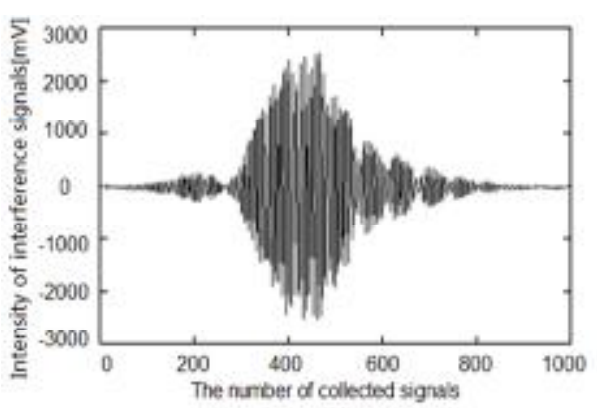

(a)

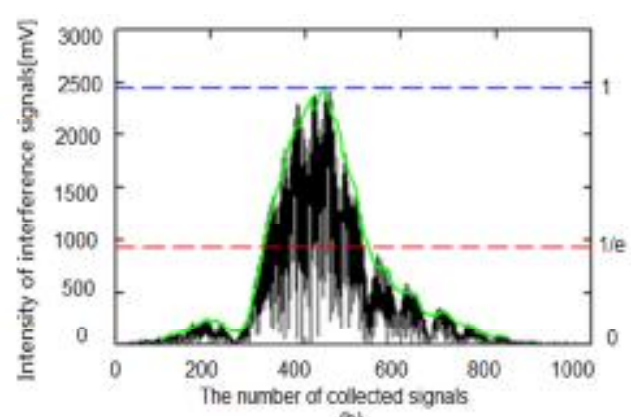

(b)

Fig.3. The method of locating signal peak.(a) The primary signals collected; (b) Envelope image of signal

The method can be described as follows: i) Firstly, all points whose values are greater than 1/e of the maximum are selected, and then these points are plotted as a curve which called the original curve, it can be seen in Fig.4(a). After that, a symmetry axis which is parallel to the vertical axis and passes through the maximum value point of the original curve is drawn, then, the original curve revolves around the symmetry axis and forms another curve which is called revolved curve. ii) The revolved curve is moved left or right along the horizontal axis. Meanwhile, the mean square error between the original curve and the revolved curve is calculated. When the mean square error obtained is the smallest, the best translational position to the revolved curve is determined. iii) The revolved curve was moved according to the translation amount, as shown in Fig.4(b). Finally, the symmetrical position of the two curves is used as the zero-path difference position. The method utilizes the least squares method can equalize the errors brought by the interference signals and the measurement error and the system error can be effectively reduced.

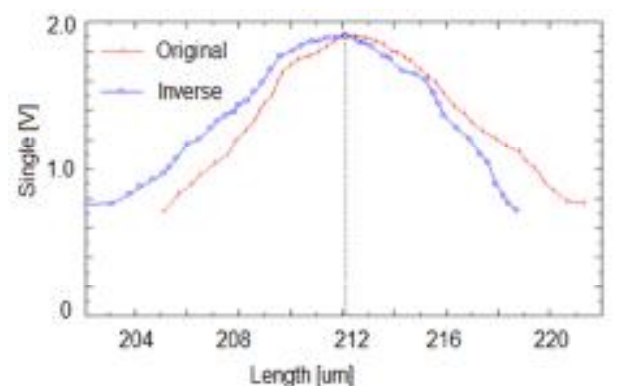

(a)

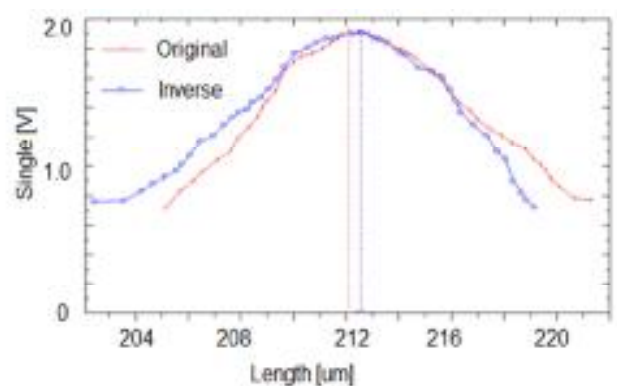

(b)

Fig.4. The method of locating wave crests.(a) Flip chart; (b) Flipping the curve after translation

\section{Specular distance measurement experiment and error analysis}

According to the design principle of lens spacing detection system, a measurement system is set up as shown in Fig.5. Among them, the center wavelength of the detection system is $1310 \mathrm{~nm}$, and the half wavelength width of the SLD wide spectrum light source is $85 \mathrm{~nm}$, and its optical power is $10.8 \mathrm{~mW}$.

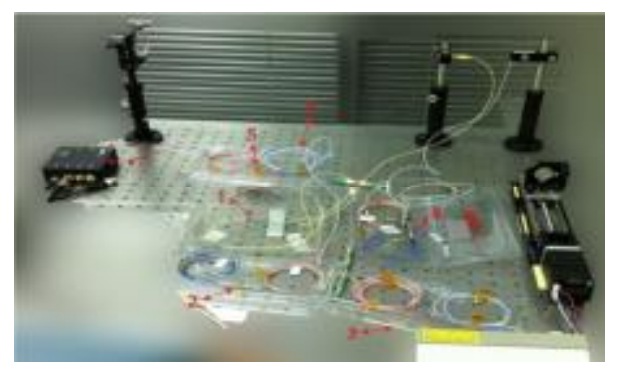

Fig.5 Measurement test system for detecting the distance between the mirror surfaces of a compound lens (1) a wave division multiplexer, (2) Circulator 1, (3) 80/20 fiber couplers, (4) Circulator 2, (5) 50/50 optical couplers, (6) the single mode fiber , (7) balance detector 
In order to test the measurement accuracy of the system designed in this article, an achromatic composite lens group is selected to perform experiment for detecting distance between the mirror surfaces. Among them, the compound lens includes 7 groups of lenses, and the structure of the combined lens is shown in Fig.6.



Fig.6 The structure of the lens group to be measured

According to the experimental system and the detection algorithm, the measurement of the lens spacing of the compound lens is carried out. The interference signal improvement is very obvious, and the output interference signal waveform was shown in Fig.7(a), and Fig.7(b) is the waveform of the interference signal after filtered.

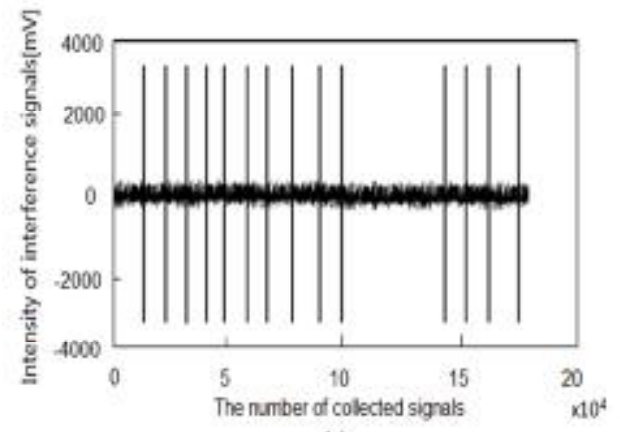

(a)

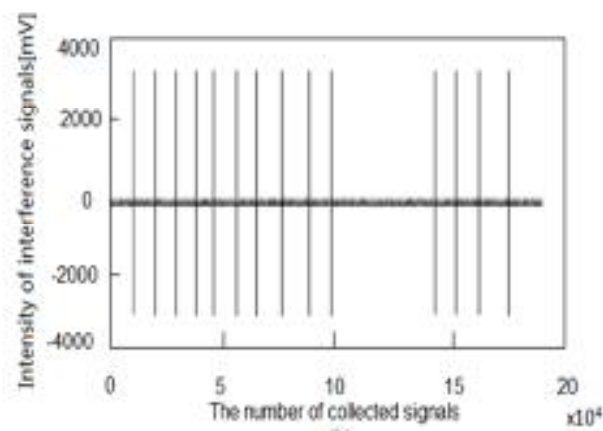

Fig.7 Measurement of the waveforms of the interferometric signal in the lens group, (a) the waveforms of the interfering signal before filtering,(b) the waveforms of the interfering signal after filtering

In order to evaluate the accuracy and stability of the test system, the lens group has been measured for many times. Table. 1 lists the design values of the measured lenses, the average values of the ten measurements and the standard deviation calculated.

Table.1 Measurement data of spacing thickness of lens group

\begin{tabular}{llll}
\hline Sample & $\begin{array}{l}\text { Design thickness } \\
\text { or spacing[mm] }\end{array}$ & $\begin{array}{l}\text { The mean value of } \\
\text { measuring thickness or } \\
\text { spacing [mm] }\end{array}$ & $\begin{array}{l}\text { Standard } \\
\text { deviation[nm] }\end{array}$ \\
\hline Lens 1 & 5.00 & 5.0327 & 59 \\
Air gap 1-2 & 6.00 & 6.0155 & 101 \\
Lens 2 & 5.00 & 4.9879 & 127 \\
Air gap 2-3 & 5.00 & 5.0214 & 167 \\
Lens 3 & 6.00 & 5.9897 & 201 \\
Air gap 3-4 & 5.90 & 5.8591 & 221 \\
Lens 4 & 6.00 & 6.0319 & 260 \\
Air gap 4-5 & 8.00 & 7.9981 & 239 \\
Lens 5 & 5.00 & 4.9794 & 327 \\
Air gap 5-6 & 18.00 & 17.8951 & 389 \\
Lens 6 & 5.00 & 5.0124 & 372 \\
Air gap 6-7 & 7.00 & 6.9978 & 408 \\
Lens 7 & 7.00 & 7.0214 & 425 \\
\hline
\end{tabular}


According to the results of the measurement data, it can be seen that the relative intensity of the reflected light on the lens surface at the rear end of the lens group is weak and the standard deviation of measurement is relatively large due to the influence of the number of lenses contained a compound lens and the reflection loss of the lens surface. With the increase of the number of lens to be measured, the interference signal gradually get weakened and the precise of the positioning accuracy of the zero optical path difference position be reduced, which will lead to an increase in the measurement standard deviation in measuring the lens spacing of the compound lens.

\section{Conclusion}

In this paper, based on the low coherent light interference method, a system for measuring the lens spacing of the compound lens is designed. A calculation method of interference signal frequency in measurement system is put forward, and a bandpass filter is designed, which can effectively improve the signal-to-noise ratio of the interferometric signal. The fitting method of the interference signal envelope is proposed. Based on the characteristics of the low-coherence interference signal, we used a least-squares correction information positioning method, which effectively improves the repeatability of the measurement. Finally, by testing the lens spacing of the combined lens, the effectiveness of the proposed design method is proved. The detection system not only is convenient, fast, and has no damage to optical devices, but also has high precision and strong stability. It has a good application prospect in optical processing and optical detection.

\section{Acknowledgements}

This work is supported by the National Natural Science Foundation of China (Grant No.51575099)

\section{References}

[1] A.V. Goncharov, L.L. Bailon and N.M. Devaney. Spie:Vol.7389(2009),p.738912.

[2] M. Kunkel, J. Schulze. Glass Science and Technology: Vol.78(2005),p.245.

[3] L.B. Shi, L.R. Qiu and Y. Wang. Chinese Journal of Scientific Instrument :Vol. 33(2012),p. 683.

[4] H.W. Gao, H. Wang, Y.Y. Liu and Y. Yu. Journal of Electronic Measurement and Instrument: Vol.31(2017),p.820.

[5] J.H. Zhang, Q Liu and R.H. Nie.Spie:Vol.36(2016),p.174.

[6] P. Langehanenberg,A. Ruprecht. Spie:Vol.8844(2013),p.88444F.

[7] J. Benitez, J. Mora. IEEE Photonics Technology Letters:Vol.29(2017),p.1735.

[8] S. Merlo, P. Poma and E. Crisa. Sensors:Vol.17(2017).

[9] O. Martinez-Matos, C. Rickenstorff and S. Zamora. Optics Express:Vol.25(2017),p.3222.

[10] R. Abuter, M. Accardo and A .Amorim. Astronomy \& Astrophysics:Vol.602(2017).

[11] R.W Kunze, R. Schmitt. Tm-Technisches Messen:Vol.84(2017),p.575.

[12] K.Li, M. Jiang, Z.Z. Zhao and Z.M.Wang. Optics Communications:Vol.389(2017),p.234.

[13] J.M.Tang, H.W. Liu, Q.F. Zhang, B.P. Ren and Y.H. Liu. IEEE Transactions on Applied Superconductivity:Vol.28(2018).

[14] H Lu, Z.Q. Yue and J.L. Zhao. Optics Communications:Vol.414(2018). 